Report no. NA-04/02

\title{
Discontinuous Galerkin methods for first-order hyperbolic problems
}

\author{
F. Brezzi $i^{1,2}$, L. D. Marini ${ }^{1,2}$, and E. Süli ${ }^{3}$
}

In this paper we consider discontinuous Galerkin (DG) finite element approximations of a model scalar linear hyperbolic equation. We show that in order to ensure continuous stabilization of the method it suffices to add a jump-penalty-term to the discretized equation. In particular, the method does not require upwinding in the usual sense. For a specific value of the penalty parameter we recover the classical discontinuous Galerkin method with upwind numerical flux function. More generally, using discontinuous piecewise polynomials of degree $k$, the familiar optimal $\mathcal{O}\left(h^{k+1 / 2}\right)$ error estimate is proved for any value of the penalty parameter. As precisely the same jump -term is used for the purposes of stabilizing DG approximations of advection-diffusion operators, the discretization proposed here can simplify the construction of discontinuous Galerkin finite element approximations of advection-diffusion problems. Moreover, the use of the jump-stabilization makes the analysis simpler and more elegant.

Oxford University Computing Laboratory

Numerical Analysis Group

Wolfson Building

Parks Road

Oxford, England OX1 3QD

January, 2004

${ }^{1}$ Dipartimento di Matematica, Università di Pavia, Via Ferrata 1, 27100 Pavia, Italy

${ }^{2}$ IMATI del CNR, Via Ferrata 1, 27100 Pavia, Italy

${ }^{3}$ University of Oxford, Computing Laboratory, Wolfson Building, Parks Road, Oxford OX1 3QD, United Kingdom 


\section{Introduction}

Let $\Omega$ be a bounded polygonal domain in $\mathbf{R}^{2}$, and let the advective velocity field $\boldsymbol{\beta}=$ $\left(\beta_{1}, \beta_{2}\right)^{\mathrm{T}}$ be a vector-valued function defined on $\bar{\Omega}$ with $\beta_{i} \in \mathrm{C}^{1}(\bar{\Omega}), i=1,2$. We define the inflow and outflow parts of $\Gamma=\partial \Omega$ in the usual fashion:

$$
\begin{aligned}
& \Gamma_{-}=\{x \in \Gamma: \boldsymbol{\beta}(x) \cdot \mathbf{n}(x)<0\}=\text { inflow } \\
& \Gamma_{+}=\{x \in \Gamma: \boldsymbol{\beta}(x) \cdot \mathbf{n}(x)>0\}=\text { outflow }
\end{aligned}
$$

where $\mathbf{n}(x)$ denotes the unit outward normal vector to $\Gamma$ at $x \in \Gamma$.

Let $\gamma \in \mathrm{C}(\bar{\Omega}), f \in \mathrm{L}^{2}(\Omega), g \in \mathrm{L}^{2}\left(\Gamma_{-}\right)$. Consider the hyperbolic boundary value problem

$$
\begin{array}{rlrl}
\mathcal{L} u \equiv \operatorname{div}(\boldsymbol{\beta} u)+\gamma u=f & & \text { in } \Omega, \\
u & =g & & \text { on } \Gamma_{-} .
\end{array}
$$

We shall assume the existence of a positive constant $c_{0}$ such that

$$
\gamma(x)+\frac{1}{2} \operatorname{div} \beta(x) \geq c_{0} \quad \text { for all } x \in \bar{\Omega} .
$$

The discontinuous Galerkin approximation of (1.1) consists of choosing the space $V_{h}^{k}$ of discontinuous piecewise polynomials of degree $k \geq 0$ and seeking $u_{h} \in V_{h}^{k}$ such that

$$
\begin{gathered}
\sum_{T \in \mathcal{T}_{h}} \int_{T}\left(-u_{h}\left(\boldsymbol{\beta} \cdot \nabla v_{h}\right)+\gamma u_{h} v_{h}\right) \mathrm{d} x+\sum_{e \not \subset \Gamma_{-}} \int_{e}\left\{\boldsymbol{\beta} u_{h}\right\}_{u} \cdot \llbracket v_{h} \rrbracket \mathrm{d} s \\
=\int_{\Omega} f v_{h} \mathrm{~d} x-\sum_{e \subset \Gamma_{-}} \int_{e}(\boldsymbol{\beta} \cdot \mathbf{n}) g v_{h} \mathrm{~d} s, \quad v_{h} \in V_{h}^{k},
\end{gathered}
$$

where $\left\{\boldsymbol{\beta} u_{h}\right\}_{u}$ represents the upwind value of $\boldsymbol{\beta} u_{h}$ and, as usual, 【 $v_{h} \rrbracket$ denotes the jump of $v_{h}$ across the edge of an element over which it is evaluated; the precise definition is given in the next section in (2.1) and (2.3).

Here we propose a slightly different stabilization of the problem. Instead of (1.3) we consider

$$
\begin{aligned}
& \sum_{T \in \mathcal{T}_{h}} \int_{T}\left(-u_{h}\left(\boldsymbol{\beta} \cdot \nabla v_{h}\right)+\gamma u_{h} v_{h}\right) \mathrm{d} x+\sum_{e \not \subset \Gamma_{-}} \int_{e}\left\{\boldsymbol{\beta} u_{h}\right\} \cdot \llbracket v_{h} \rrbracket \mathrm{d} s \\
& +\sum_{e \not \subset \Gamma} \int_{e} c_{e}(s) \llbracket u_{h} \rrbracket \cdot \llbracket v_{h} \rrbracket \mathrm{d} s=\int_{\Omega} f v_{h} \mathrm{~d} x-\sum_{e \subset \Gamma_{-}} \int_{e}(\boldsymbol{\beta} \cdot \mathbf{n}) g v_{h} \mathrm{~d} s, \quad v_{h} \in V_{h}^{k},
\end{aligned}
$$

where $\left\{\boldsymbol{\beta} u_{h}\right\}$ is now the usual average (see (2.2)) and, for every internal edge $e$, we denoted by $c_{e}$ a nonnegative function to be chosen (which, in practical implementations, could be defined as constant on $e$ ). For related ideas concerning least-squares-type stabilization in the context of discontinuous Galerkin methods, we refer to Section 5 of the paper [5].

We shall prove that, when the stabilization function is taken to be $c_{e}=|\boldsymbol{\beta} \cdot \mathbf{n}| / 2$ then (1.4) collapses to the original discontinuous Galerkin method (1.3). This fact is 
essentially known, and has already been used, for instance, by Cockburn and Shu [4] in selecting the numerical flux functions for LDG methods in order to reduce the stencil. However, the discontinuous Galerkin method (1.4) with jump-stabilization is stable more generally, whenever there exists a $\theta_{0}>0$ such that

$$
c_{e} \geq \theta_{0}\left|\boldsymbol{\beta} \cdot \mathbf{n}_{e}\right| \quad \text { for each internal edge } e \text {. }
$$

This approach, in our opinion, has several potential advantages. In the first place we have a way to tune-up the amount of upwinding that we are willing to use. Admittedly, this is a rather insignificant advantage for a problem as simple as our model problem (1.1); however, the technique can be relevant in more complicated situations: for instance, in the case of advection-diffusion equations where a certain amount of viscosity is present, possibly only in subsets of the computational domain, particularly when such subsets are unknown a priori, or change with time, or when it is necessary to alter the amount of local numerical dissipation in the course of an iterative procedure. In fact, if a diffusive term is present and is also approximated by means of a discontinuous Galerkin method, it is quite likely that a jump-penalty, identical or very similar to the present one, is already included into the diffusive part of the discretization, and we can therefore treat the two jump-penalty stabilizations together, both from the theoretical viewpoint as well as in the actual implementation of the method. Finally, we believe that the present way of dealing with upwinding provides a simpler and more elegant analysis even in the case when we take exactly $c_{e} \equiv|\boldsymbol{\beta} \cdot \mathbf{n}| / 2$.

The paper is organized as follows. In the next section we formulate our hypotheses, and we derive the unstabilized discontinuous Galerkin method for our model problem. Then, in Section 3, we introduce the jump-stabilization and arrive at the ultimate form of our method. The consistency and the stability of the method are explored in Section 4 , and the final a priori error estimates are proved in Section 5. In particular, in the case of discontinuous piecewise polynomial finite element approximations of degree $k \geq 0$, we prove an optimal error estimate of the form

$$
\left\|u-u_{h}\right\|_{0, \Omega}^{2}+\sum_{e \in \mathcal{E}_{h}}\left\|c_{e}^{1 / 2} \llbracket u-u_{h} \rrbracket\right\|_{0, e}^{2} \leq C h^{2 k+1}\|u\|_{k+1, \Omega}^{2}
$$

familiar from the theory of stabilized finite element methods for first-order hyperbolic problems.

\section{The discontinuous finite element approximation}

Let $\mathcal{T}_{h}$ be a regular family of decompositions of $\Omega$ into triangles $T$; let $h_{T}$ denote the diameter of $T$, and let $h=\max _{T \in \mathcal{T}_{h}} h_{T}$. In order to define a discontinuous finite element approximation of problem (1.1) we first need to introduce typical tools such as jumps and averages of scalar- and vector-valued functions across the edges of $\mathcal{T}_{h}$. Following the notation of [3], let $e$ be an interior edge shared by elements $T_{1}$ and $T_{2}$. Define the unit normal vectors $\mathbf{n}^{1}$ and $\mathbf{n}^{2}$ on $e$ pointing exterior to $T_{1}$ and $T_{2}$, respectively. For a 
function $\varphi$, piecewise smooth on $\mathcal{T}_{h}$, with $\varphi^{i}:=\left.\varphi\right|_{T_{i}}$ we define

$$
\{\varphi\}=\frac{1}{2}\left(\varphi^{1}+\varphi^{2}\right), \quad \llbracket \varphi \rrbracket=\varphi^{1} \mathbf{n}^{1}+\varphi^{2} \mathbf{n}^{2} \quad \text { on } e \in \mathcal{E}_{h}^{\circ}
$$

where $\mathcal{E}_{h}^{\circ}$ is the set of interior edges $e$. For a vector-valued function $\boldsymbol{\tau}$, piecewise smooth on $\mathcal{T}_{h}$, with analogous meaning for $\boldsymbol{\tau}^{1}$ and $\boldsymbol{\tau}^{2}$, we define

$$
\{\boldsymbol{\tau}\}=\frac{1}{2}\left(\boldsymbol{\tau}^{1}+\boldsymbol{\tau}^{2}\right), \quad \llbracket \boldsymbol{\tau} \rrbracket=\boldsymbol{\tau}^{1} \cdot \mathbf{n}^{1}+\boldsymbol{\tau}^{2} \cdot \mathbf{n}^{2} \quad \text { on } e \in \mathcal{E}_{h}^{\circ}
$$

Notice that the jump $\varphi \varphi \rrbracket$ of the scalar function $\varphi$ across $e \in \mathcal{E}_{h}^{\circ}$ is a vector parallel with the normal to $e$, and the jump $\llbracket \boldsymbol{\tau} \rrbracket$ of the vector function $\boldsymbol{\tau}$ is a scalar quantity. The advantage of these definitions is that they do not depend on the ordering that is assigned to the elements $T_{i}$. For $e \in \mathcal{E}_{h}^{\partial}$, the set of boundary edges, we let

$$
\llbracket \varphi \rrbracket=\varphi \mathbf{n}, \quad\{\boldsymbol{\tau}\}=\boldsymbol{\tau} \quad \text { on } e \in \mathcal{E}_{h}^{\partial} .
$$

We do not require either of the quantities $\{\varphi\}$ or $\llbracket \boldsymbol{\tau} \rrbracket$ on boundary edges, and leave them undefined there.

Next, with any integer $k \geq 0$ we associate the finite element space of discontinuous piecewise polynomial functions

$$
V_{h}^{k}=\left\{v \in \mathrm{L}^{2}(\Omega): v_{\mid T} \in \mathrm{P}_{k}(T) \quad T \in \mathcal{T}_{h}\right\},
$$

where, as usual, $\mathrm{P}_{k}$ is the space of polynomials of degree $k$ or less. On multiplying equation (1.1) by a function $v_{h} \in V_{h}^{k}$ and integrating by parts, we get

$$
\sum_{T \in \mathcal{T}_{h}}\left(\int_{T}\left(-u\left(\boldsymbol{\beta} \cdot \nabla v_{h}\right)+\gamma u v_{h}\right) \mathrm{d} x+\int_{\partial T}(\boldsymbol{\beta} \cdot \mathbf{n}) u v_{h} \mathrm{~d} s\right)=\int_{\Omega} f v_{h} \mathrm{~d} x .
$$

Recall the following identity (see [3]) which holds for vectors $\boldsymbol{\tau}$ and scalars $\varphi$, piecewise smooth on $\mathcal{T}_{h}$ :

$$
\sum_{T \in \mathcal{T}_{h}} \int_{\partial T}(\boldsymbol{\tau} \cdot \mathbf{n}) \varphi \mathrm{d} s=\sum_{e \in \mathcal{E}_{h}} \int_{e}\{\boldsymbol{\tau}\} \cdot \llbracket \varphi \rrbracket \mathrm{d} s+\sum_{e \in \mathcal{E}_{h}^{\circ}} \int_{e} \llbracket \boldsymbol{\tau} \rrbracket\{\varphi\} \mathrm{d} s .
$$

Now, from (2.6) with $\boldsymbol{\tau}=\boldsymbol{\beta} u$ and $\varphi=v_{h}$, since $\llbracket \boldsymbol{\beta} u \rrbracket=0$ on internal edges, we deduce that

$$
\begin{aligned}
\sum_{T \in \mathcal{T}_{h}} \int_{\partial T}(\boldsymbol{\beta} \cdot \mathbf{n}) u v_{h} \mathrm{~d} s & =\sum_{e \in \mathcal{E}_{h}} \int_{e}\{\boldsymbol{\beta} u\} \cdot \llbracket v_{h} \rrbracket \mathrm{d} s \\
& =\sum_{e \not \subset \Gamma_{-}} \int_{e}\{\boldsymbol{\beta} u\} \cdot \llbracket v_{h} \rrbracket \mathrm{d} s+\sum_{e \subset \Gamma_{-}} \int_{e} \boldsymbol{\beta} \cdot \mathbf{n} g v_{h} \mathrm{~d} s .
\end{aligned}
$$

Setting

$$
a_{h}\left(u, v_{h}\right)=\sum_{T \in \mathcal{T}_{h}} \int_{T}\left(-u\left(\boldsymbol{\beta} \cdot \nabla v_{h}\right)+\gamma u v_{h}\right) \mathrm{d} x
$$




$$
\begin{gathered}
b_{h}\left(u, v_{h}\right)=\sum_{e \not \subset \Gamma_{-}} \int_{e}\{\boldsymbol{\beta} u\} \cdot \llbracket v_{h} \rrbracket \mathrm{d} s, \\
\left(f, v_{h}\right)=\int_{\Omega} f v_{h} \mathrm{~d} x, \quad\left\langle g, v_{h}\right\rangle=-\sum_{e \subset \Gamma_{-}} \int_{e}(\boldsymbol{\beta} \cdot \mathbf{n}) g v_{h} \mathrm{~d} s,
\end{gathered}
$$

we then have that

$$
a_{h}\left(u, v_{h}\right)+b_{h}\left(u, v_{h}\right)=\left(f, v_{h}\right)+\left\langle g, v_{h}\right\rangle, \quad v_{h} \in V_{h}^{k}
$$

Inspired by the identity (2.11), we now define the unstabilized discrete problem as

$$
\left\{\begin{array}{l}
\text { find } u_{h} \in V_{h}^{k}: \\
a_{h}\left(u_{h}, v_{h}\right)+b_{h}\left(u_{h}, v_{h}\right)=\left(f, v_{h}\right)+\left\langle g, v_{h}\right\rangle, \quad v_{h} \in V_{h}^{k} .
\end{array}\right.
$$

\section{$3 \quad$ Stabilization with a jump-penalty}

The formulation (2.12) is stable, but only in the $\mathrm{L}^{2}(\Omega)$-norm. The practical consequences of this can be detrimental: discontinuities in the boundary data may trigger large, nonphysical oscillations in the numerical solution. In order to design a formulation that is stable in a stronger norm, on every internal edge $e$, common to the triangles $T^{1}$ and $T^{2}$, one usually substitutes the average $\left\{\boldsymbol{\beta} u_{h}\right\}$ that appears in $b_{h}\left(u_{h}, v_{h}\right)$ (see (2.9)) by the upwind value of $\boldsymbol{\beta} u_{h}$, defined as

$$
\left\{\boldsymbol{\beta} u_{h}\right\}_{u}= \begin{cases}\boldsymbol{\beta} u_{h}^{1} & \text { if } \boldsymbol{\beta} \cdot \mathbf{n}^{1}>0 \\ \boldsymbol{\beta} u_{h}^{2} & \text { if } \boldsymbol{\beta} \cdot \mathbf{n}^{1}<0 \\ \boldsymbol{\beta}\left\{u_{h}\right\} & \text { if } \boldsymbol{\beta} \cdot \mathbf{n}^{1}=0\end{cases}
$$

As $\left\{\boldsymbol{\beta} u_{h}\right\}_{u}$, in $b_{h}\left(u_{h}, v_{h}\right)$, is multiplied by $\llbracket v_{h} \rrbracket$, which is directed as the normal $\mathbf{n}$ to $e$, it is clear that only the normal component of $\left\{\boldsymbol{\beta} u_{h}\right\}_{u}$ will feature in the scheme.

On the other hand, it is a simple matter to check that, if $\mathbf{n}$ is normal to $e$, then $\left\{\boldsymbol{\beta} u_{h}\right\}_{u} \cdot \mathbf{n}$ can also be written as

$$
\left\{\boldsymbol{\beta} u_{h}\right\}_{u} \cdot \mathbf{n}=\left(\left\{\boldsymbol{\beta} u_{h}\right\}+c^{*} \llbracket u_{h} \rrbracket\right) \cdot \mathbf{n}
$$

where $\left\{\boldsymbol{\beta} u_{h}\right\}$ is again the usual average and $c^{*}$ is given by

$$
c^{*}=|\boldsymbol{\beta} \cdot \mathbf{n}| / 2 \text {. }
$$

Motivated by (3.2) and (3.3), we now hypothesize (and will prove later on) that we could still achieve stability in a norm that is stronger than $\|\cdot\|_{0, \Omega}$ if we replace the upwind average $\left\{\boldsymbol{\beta} u_{h}\right\}_{u}$ by $\left\{\boldsymbol{\beta} u_{h}\right\}+c_{e} \llbracket u_{h} \rrbracket$, provided that $c_{e}$ is a nonnegative function chosen on each $e$ in such a way that

$$
c_{e} \geq \theta_{0}|\boldsymbol{\beta} \cdot \mathbf{n}|
$$


with $\theta_{0}$ a positive constant independent of $e$ and $h$. In order to make our proofs more elegant, it will be convenient to define $c_{e}$ on the boundary $\partial \Omega$ as well by setting

$$
c_{e}=\left\{\begin{aligned}
\boldsymbol{\beta} \cdot \mathbf{n}_{\Omega} / 2 & \text { on } \Gamma_{+} \\
-\boldsymbol{\beta} \cdot \mathbf{n}_{\Omega} / 2 & \text { on } \Gamma_{-},
\end{aligned}\right.
$$

where $\mathbf{n}_{\Omega}$ is the unit normal vector to $\partial \Omega$.

We emphasize here that, trivially, the conditions (3.4) and (3.5) imply that

$$
c_{e} \geq 0 \quad \text { for all } e \in \mathcal{E}_{h} \text {. }
$$

We therefore assume that $c_{e}$ satisfies (3.4) and we replace $b_{h}\left(u_{h}, v_{h}\right)$ in (2.12) by its stabilized version

$$
b_{h}^{\mathrm{s}}\left(u_{h}, v_{h}\right):=b_{h}\left(u_{h}, v_{h}\right)+\sum_{e \in \mathcal{E}_{h}^{\circ}} \int_{e} c_{e} \llbracket u_{h} \rrbracket \cdot \llbracket v_{h} \rrbracket \mathrm{d} s,
$$

thus obtaining the stabilized discrete problem

$$
\left\{\begin{array}{l}
\text { find } u_{h} \in V_{h}^{k}: \\
a_{h}\left(u_{h}, v_{h}\right)+b_{h}^{\mathrm{s}}\left(u_{h}, v_{h}\right)=\left(f, v_{h}\right)+\left\langle g, v_{h}\right\rangle, \quad v_{h} \in V_{h}^{k} .
\end{array}\right.
$$

We note, in particular, that (3.8) includes, as a special case, the classical discontinuous Galerkin finite element method (see, [7] and [8]) with the numerical flux function taken as the upwind flux. Indeed, we can always choose $c_{e}=c^{*}$, given by (3.3).

We also note that in certain cases taking $\left\{\boldsymbol{\beta} u_{h}\right\}+c_{e} \llbracket u_{h} \rrbracket$ instead of the usual average corresponds to taking a different type of average. To see this, consider an internal edge $e$ (common to the triangles $T_{1}$ and $T_{2}$ ) and assume in particular that the function $c_{e}$ vanishes whenever $\boldsymbol{\beta} \cdot \mathbf{n}_{e}$ does. In this case we can define, for $i=1,2$,

$$
\alpha^{i}= \begin{cases}\frac{1}{2}+\frac{c_{e}}{\boldsymbol{\beta} \cdot \mathbf{n}^{i}} & \text { if } \boldsymbol{\beta} \cdot \mathbf{n}^{i} \neq 0 \\ \frac{1}{2} & \text { if } \boldsymbol{\beta} \cdot \mathbf{n}^{i}=0 .\end{cases}
$$

Clearly, $\alpha^{1}+\alpha^{2}=1$. We can, therefore, define the tilted average

$$
\left\{\boldsymbol{\beta} u_{h}\right\}_{\alpha}:=\boldsymbol{\beta}\left(u_{h}^{1} \alpha^{1}+u_{h}^{2} \alpha^{2}\right) .
$$

It then follows that, whenever $\mathbf{n}_{e}$ is orthogonal to $e$, we have that

$$
\left(\left\{\boldsymbol{\beta} u_{h}\right\}+c_{e} \llbracket u_{h} \rrbracket\right) \cdot \mathbf{n}_{e}=\left\{\boldsymbol{\beta} u_{h}\right\}_{\alpha} \cdot \mathbf{n}_{e} .
$$

Therefore our jump-stabilization could also be seen as using the tilted average (3.10) instead of the usual average. 


\section{Consistency and stability of the method}

Consistency. Consistency follows immediately from (2.11) and (3.7) upon observing that, since $\boldsymbol{\beta} \cdot \llbracket u \rrbracket=0$ on internal edges, $b_{h}^{\mathrm{s}}\left(u, v_{h}\right) \equiv b_{h}\left(u, v_{h}\right)$. In particular, Galerkin orthogonality holds:

$$
a_{h}\left(u-u_{h}, v_{h}\right)+b_{h}^{\mathrm{s}}\left(u-u_{h}, v_{h}\right)=0, \quad v_{h} \in V_{h}^{k} .
$$

Stability. We shall prove stability and error estimates in the norm

$$
\|\cdot\|=\left(\|\cdot\|_{0, \Omega}^{2}+\sum_{e \in \mathcal{E}_{h}}\left\|c_{e}^{1 / 2} \llbracket \cdot \rrbracket\right\|_{0, e}^{2}\right)^{1 / 2} .
$$

The norm (4.2) is well defined on $H^{1}(\Omega)+V_{h}^{k}$, thanks to (3.6). After integration by parts, the definition $(2.8)$ of $a_{h}(\cdot, \cdot)$ yields that

$$
a_{h}\left(v_{h}, v_{h}\right)=\sum_{T \in \mathcal{T}_{h}} \int_{T}\left(\frac{1}{2} \operatorname{div} \boldsymbol{\beta}+\gamma\right) v_{h}^{2} \mathrm{~d} x-\frac{1}{2} \sum_{T \in \mathcal{T}_{h}} \int_{\partial T}(\boldsymbol{\beta} \cdot \mathbf{n}) v_{h}^{2} \mathrm{~d} s .
$$

Furthermore, from (2.6) with $\boldsymbol{\tau}=\boldsymbol{\beta}$ and $\varphi=v_{h}^{2}$, since $\llbracket \boldsymbol{\beta} \rrbracket=0$, we have

$$
\begin{aligned}
\sum_{T \in \mathcal{T}_{h}} \int_{\partial T}(\boldsymbol{\beta} \cdot \mathbf{n}) v_{h}^{2} \mathrm{~d} s & =\sum_{e \in \mathcal{E}_{h}} \int_{e}\{\boldsymbol{\beta}\} \cdot \llbracket v_{h}^{2} \rrbracket \mathrm{d} s \\
& =\sum_{e \in \mathcal{E}_{h}^{\circ}} \int_{e}\{\boldsymbol{\beta}\} \cdot \llbracket v_{h}^{2} \rrbracket \mathrm{d} s+\sum_{e \in \mathcal{E}_{h}^{\partial}} \int_{e}\{\boldsymbol{\beta}\} \cdot \llbracket v_{h}^{2} \rrbracket \mathrm{d} s .
\end{aligned}
$$

Combining (4.3) and (4.4), and splitting the contributions on $\mathcal{E}_{h}^{\partial}$ into their parts on $\Gamma_{+}$ and $\Gamma_{-}$we can then write

$$
\begin{aligned}
a_{h}\left(v_{h}, v_{h}\right)= & \sum_{T \in \mathcal{T}_{h}} \int_{T}\left(\frac{1}{2} \operatorname{div} \boldsymbol{\beta}+\gamma\right) v_{h}^{2} \mathrm{~d} x-\frac{1}{2} \sum_{e \in \mathcal{E}_{h}^{\circ}} \int_{e}\{\boldsymbol{\beta}\} \cdot \llbracket v_{h}^{2} \rrbracket \mathrm{d} s \\
& -\frac{1}{2} \sum_{e \subset \Gamma_{+}} \int_{e}\{\boldsymbol{\beta}\} \cdot \llbracket v_{h}^{2} \rrbracket \mathrm{d} s-\frac{1}{2} \sum_{e \subset \Gamma_{-}} \int_{e}\{\boldsymbol{\beta}\} \cdot \llbracket v_{h}^{2} \rrbracket \mathrm{d} s .
\end{aligned}
$$

On the other hand, using the continuity of $\boldsymbol{\beta}$ and the definitions of averages and jumps (2.1), (2.2), and (2.3), we have that

$$
\left\{\boldsymbol{\beta} v_{h}\right\} \cdot \llbracket v_{h} \rrbracket \equiv\{\boldsymbol{\beta}\} \llbracket v_{h}^{2} \rrbracket .
$$

Formula (4.6) is straightforward, but crucial. Its validity allows a simpler treatment of the jump-stabilization (where the usual average still appears explicitly), compared with the classical upwind stabilization. Indeed from (4.6) we immediately have

$$
\begin{aligned}
b_{h}\left(v_{h}, v_{h}\right) & =\sum_{e \in \mathcal{E}_{h}^{\circ}} \int_{e}\left\{\boldsymbol{\beta} v_{h}\right\} \cdot \llbracket v_{h} \rrbracket \mathrm{d} s+\sum_{e \subset \Gamma_{+}} \int_{e}\left\{\boldsymbol{\beta} v_{h}\right\} \cdot \llbracket v_{h} \rrbracket \mathrm{d} s \\
& =\frac{1}{2} \sum_{e \in \mathcal{E}_{h}^{\circ}} \int_{e}\{\boldsymbol{\beta}\} \cdot \llbracket v_{h}^{2} \rrbracket \mathrm{d} s+\sum_{e \subset \Gamma_{+}} \int_{e}\{\boldsymbol{\beta}\} \cdot \llbracket v_{h}^{2} \rrbracket \mathrm{d} s .
\end{aligned}
$$


Consequently, using (4.7) and (3.7) with (3.4) we obtain

$$
\begin{aligned}
b_{h}^{\mathrm{s}}\left(v_{h}, v_{h}\right)= & \frac{1}{2} \sum_{e \in \mathcal{E}_{h}^{\circ}} \int_{e}\{\boldsymbol{\beta}\} \cdot \llbracket v_{h}^{2} \rrbracket \mathrm{d} s \\
& +\sum_{e \subset \Gamma_{+}} \int_{e}\{\boldsymbol{\beta}\} \cdot \llbracket v_{h}^{2} \rrbracket \mathrm{d} s+\sum_{e \in \mathcal{E}_{h}^{\circ}} \int_{e} c_{e}\left|\llbracket v_{h} \rrbracket\right|^{2} \mathrm{~d} s .
\end{aligned}
$$

Finally, we note that the conditions on the boundary (3.5) and (2.3) imply that

$$
c_{e}\left|\llbracket v_{h} \rrbracket\right|^{2}=\left\{\begin{array}{rll}
\{\boldsymbol{\beta}\} \cdot \llbracket v_{h}^{2} \rrbracket / 2 & \text { on } & \Gamma_{+} \\
-\{\boldsymbol{\beta}\} \cdot \llbracket v_{h}^{2} \rrbracket / 2 & \text { on } & \Gamma_{-} .
\end{array}\right.
$$

Collecting (4.5) and (4.8), using (4.9), then (1.2) and (3.6), and finally (4.2), we obtain

$$
\begin{aligned}
a_{h}\left(v_{h}, v_{h}\right)+ & b_{h}^{\mathrm{s}}\left(v_{h}, v_{h}\right) \\
= & \sum_{T \in \mathcal{T}_{h}} \int_{T}\left(\frac{1}{2} \operatorname{div} \boldsymbol{\beta}+\gamma\right) v_{h}^{2} \mathrm{~d} x-\frac{1}{2} \sum_{e \subset \Gamma_{-}} \int_{e}\{\boldsymbol{\beta}\} \cdot \llbracket v_{h}^{2} \rrbracket \mathrm{d} s \\
& +\frac{1}{2} \sum_{e \subset \Gamma_{+}} \int_{e}\{\boldsymbol{\beta}\} \cdot \llbracket v_{h}^{2} \rrbracket \mathrm{d} s+\sum_{e \in \mathcal{E}_{h}^{\circ}} \int_{e} c_{e}\left|\llbracket v_{h} \rrbracket\right|^{2} \mathrm{~d} s \\
= & \sum_{T \in \mathcal{T}_{h}} \int_{T}\left(\frac{1}{2} \operatorname{div} \boldsymbol{\beta}+\gamma\right) v_{h}^{2} \mathrm{~d} x+\sum_{e \in \mathcal{E}_{h}} \int_{e} c_{e}\left|\llbracket v_{h} \rrbracket\right|^{2} \mathrm{~d} s \\
\geq & c_{0}\left\|v_{h}\right\|_{0, \Omega}^{2}+\sum_{e \in \mathcal{E}_{h}}\left\|c_{e}^{1 / 2} \llbracket v_{h} \rrbracket\right\|_{0, e}^{2} \geq C_{S}\left\|v_{h}\right\| \|^{2},
\end{aligned}
$$

with $C_{S}:=\min \left\{c_{0}, 1\right\}$.

\section{A priori error estimates}

In what follows $C$ will denote a generic positive constant which depends only on the degree $k$ of the polynomials, on the minimum angle of the mesh, and on the maximum value of the stabilizing functions $c_{e}$.

Let $P_{h}^{k}$ be the $\mathrm{L}^{2}$-projector onto $V_{h}^{k}$, for which the following standard estimate holds

$$
\left\|u-P_{h}^{k} u\right\|_{r, p, T} \leq C h^{k+1-r}\|u\|_{k+1, p, T}, \quad r=0,1, \quad 1 \leq p \leq \infty, \quad T \in \mathcal{T}_{h} .
$$

We recall the following trace inequality (see [1], [2]):

$$
\left\|u-P_{h}^{k} u\right\|_{0, e}^{2} \leq C\left(|e|^{-1}\left\|u-P_{h}^{k} u\right\|_{0, T}^{2}+\left|e \| u-P_{h}^{k} u\right|_{1, T}^{2}\right),
$$

with $C$ a positive constant depending only on the minimum angle of $T$. Thus, from (5.1)-(5.2) we deduce that

$$
\left\|u-P_{h}^{k} u\right\|_{0, e} \leq C h_{T}^{k+1 / 2}\|u\|_{k+1, T}, \quad e \in \mathcal{E}_{h} .
$$


Let us define

$$
\eta=u-P_{h}^{k} u, \quad \delta=u_{h}-P_{h}^{k} u .
$$

Hence from (4.10) and (4.1) we have that

$$
C_{S}\left|\|\delta \mid\|^{2} \leq a_{h}(\delta, \delta)+b_{h}^{\mathrm{s}}(\delta, \delta)=a_{h}(\eta, \delta)+b_{h}^{\mathrm{s}}(\eta, \delta) .\right.
$$

Next, observe that $\nabla \delta \in V_{h}^{k}$, so that, by the definition of the projector $P_{h}^{0}$,

$$
\int_{T}\left(P_{h}^{0} \boldsymbol{\beta} \cdot \nabla \delta\right) \eta \mathrm{d} x=0
$$

Using this, together with (5.1), the Cauchy-Schwarz inequality and the inverse inequality, we deduce that

$$
\begin{aligned}
a_{h}(\eta, \delta) & =\sum_{T \in \mathcal{T}_{h}} \int_{T}(-\eta(\boldsymbol{\beta} \cdot \nabla \delta)+\gamma \delta \eta) \mathrm{d} x \\
& \left.=\sum_{T \in \mathcal{T}_{h}} \int_{T}\left(\left(P_{h}^{0} \boldsymbol{\beta}-\boldsymbol{\beta}\right) \cdot \nabla \delta\right) \eta+\gamma \delta \eta\right) \mathrm{d} x \\
& \leq C\left(\sum_{T \in \mathcal{T}_{h}}\left\|P_{h}^{0} \boldsymbol{\beta}-\boldsymbol{\beta}\right\|_{0, \infty, T}|\delta|_{1, T}+\|\delta\|_{0, T}\right)\|\eta\|_{0, T} \\
& \leq C\left(\sum_{T \in \mathcal{T}_{h}} h_{T}|\boldsymbol{\beta}|_{1, \infty, T} h_{T}^{-1}\|\delta\|_{0, T}+\|\delta\|_{0, T}\right)\|\eta\|_{0, T} \\
& \leq C h^{k+1}\|\delta\|_{0, \Omega}\|u\|_{k+1, \Omega} .
\end{aligned}
$$

It remains to estimate $b_{h}^{\mathrm{s}}(\eta, \delta)$. For this purpose, we first make use of $(3.4)$ and the continuity of $\boldsymbol{\beta}$ to obtain, for every edge $e$ and for every unit vector $\mathbf{n}$ normal to $e$,

$$
|\{\boldsymbol{\beta} \eta\} \cdot \mathbf{n}|=|\boldsymbol{\beta} \cdot \mathbf{n}||\{\eta\}| \leq \frac{c_{e}}{\theta_{0}}|\{\eta\}| .
$$

Making use of the fact that $\llbracket \delta \rrbracket$ is also normal to $e$, and using (3.6) once again, we then have that

$$
\int_{e}\{\boldsymbol{\beta} \eta\} \cdot \llbracket \delta \rrbracket \mathrm{d} s \leq \frac{1}{\theta_{0}}\left\|c_{e}^{1 / 2}\{\eta\}\right\|_{0, e}\left\|c_{e}^{1 / 2} \llbracket \delta \rrbracket\right\|_{0, e} .
$$

Next,

$$
\int_{e} c_{e} \llbracket \eta \rrbracket \cdot \llbracket \delta \rrbracket \mathrm{d} s \leq\left\|c_{e}^{1 / 2} \llbracket \eta \rrbracket\right\|_{0, e}\left\|c_{e}^{1 / 2} \llbracket \delta \rrbracket\right\|_{0, e} .
$$

Inserting (5.8) and (5.9) into the definition (3.7) of $b_{h}^{\mathrm{s}}$, and then using the CauchySchwarz inequality and (5.3), it follows that

$$
\begin{aligned}
b_{h}^{\mathrm{s}}(\eta, \delta) & \leq \sum_{e \in \mathcal{E}_{h}}\left(\frac{1}{\theta_{0}}\left\|c_{e}^{1 / 2}\{\eta\}\right\|_{0, e}+\left\|c_{e}^{1 / 2} \llbracket \eta \rrbracket\right\|_{0, e}\right)\left\|c_{e}^{1 / 2} \llbracket \delta \rrbracket\right\|_{0, e} \\
& \leq C h^{k+1 / 2}\|u\|_{k+1, \Omega}\left(\sum_{e \in \mathcal{E}_{h}}\left\|c_{e}^{1 / 2} \llbracket \delta \rrbracket\right\|_{0, e}^{2}\right)^{1 / 2} .
\end{aligned}
$$


Substituting (5.6) and (5.10) in (5.4), and using the definition (4.2) we obtain

$$
C_{S}\|\delta\|^{2} \leq C h^{k+1 / 2}\|u\|_{k+1, \Omega}\|\delta\| \|
$$

which implies immediately that

$$
\|\delta\| \leq C h^{k+1 / 2}\|u\|_{k+1, \Omega}
$$

Hence

$$
\left\|u-u_{h}\right\| \mid \leq C h^{k+1 / 2}
$$

thus completing the error analysis of the method.

\section{Conclusions}

We considered discontinuous Galerkin finite element approximations of a model scalar linear hyperbolic equation $\operatorname{div}(\boldsymbol{\beta} u)+\gamma u=f$ in $\Omega \subset \mathbb{R}^{2}$, subject to nonhomogeneous boundary condition $u=g$ at the inflow part of $\partial \Omega$. We showed that in order to ensure continuous stabilization of the method it suffices to add a jump-penalty term to the discretized equation. A particular value of the penalty functions $c_{e}$ results in the standard upwind scheme, but, using discontinuous piecewise polynomials of degree $k$, an optimal $\mathcal{O}\left(h^{k+1 / 2}\right)$ error estimate was proved for any choice of penalty functions $c_{e}$ that satisfy $c_{e} \geq \theta_{0}\left|\boldsymbol{\beta} \cdot \mathbf{n}_{e}\right|$ with $\theta_{0}>0$ independent of $e$ and $h$. The latter property can be easily ensured by simply choosing the penalty function as a suitable constant on each edge. As precisely the same jump-term is used for stabilizing DG approximations of diffusion operators, the discretization proposed here can simplify the analysis and the implementation of discontinuous Galerkin finite element approximations of advectiondiffusion problems.

If the jump-penalty terms are omitted from the scheme by formally setting $c_{e} \equiv 0$ on each $e \in \mathcal{E}_{h}^{\circ}$, then the scheme (3.8) collapses to (2.12). Since the latter is only stable in the $\mathrm{L}^{2}(\Omega)$-norm, in the error analysis of (2.12) one is forced to use the inverse inequality

$$
\|\llbracket \delta \rrbracket\|_{0, e} \leq C h_{e}^{-1 / 2}\|\delta\|_{0, T^{1} \cup T^{2}}
$$

to revert from the edgewise $\mathrm{L}^{2}$-norm to the elemental $\mathrm{L}^{2}$-norm in the course of bounding the left-hand side of (5.8). Hence, instead of (5.8) one has

$$
\int_{e}\{\boldsymbol{\beta} \eta\} \cdot \llbracket \delta \rrbracket \mathrm{d} s \leq C\|\beta\|_{0, \infty, e}\|\{\eta\}\|_{0, e} h_{e}^{-1 / 2}\|\delta\|_{0, T^{1} \cup T^{2}} \leq C h^{k}\|\delta\|_{0, T^{1} \cup T^{2}}
$$

where $T^{1}$ and $T^{2}$ are the two triangles whose common edge is $e$; the inequality (6.1), in turn, results in the suboptimal error bound $\left\|u-u_{h}\right\|_{0, \Omega} \leq C h^{k}$ for (2.12), - in sharp contrast with the optimal-order error bound (1.6) for the stabilised scheme (3.8). This undesirable loss of optimality of the unstabilised scheme (2.12) further highlights the helpful role played in the stabilized scheme (3.8) by the jump-penalty terms. 


\section{References}

[1] S. Agmon, Lectures on Elliptic Boundary Value Problems, Van Nostrand Mathematical Studies, Princeton, NJ, 1965.

[2] D. N. ARnold, An interior penalty finite element method with discontinuous element, SIAM J. Numer. Anal., 19 (1982), pp. 742-760.

[3] D.N. Arnold, F. Brezzi, B. Cockburn, and L.D. Marini, Unified analysis of discontinuous Galerkin methods for elliptic problems, SIAM J. Numer. Anal., 39 (2002), pp. 1749-1779.

[4] B. Cockburn And C.W. Shu, The local discontinuous Galerkin finite element method for convection-diffusion systems, SIAM J. Numer. Anal., 35 (1998), pp. 2440-2463.

[5] P. Houston, M. Jensen, And E. Süli, $h p$-Discontinuous Galerkin finite element methods with least-squares stabilization, Journal of Scientific Computing, 17 (2002), pp. $1-26$.

[6] P. Houston, C. Schwab, And E. Süli, Stabilized hp-finite element methods for first-order hyperbolic problems, SIAM J. Numer. Anal., 37 (2000), pp. 1618-1643.

[7] C. Johnson And J. Pitkäranta, An analysis of the discontinuous Galerkin method for a scalar hyperbolic conservation law, Math. Comp., 46 (1986), pp.1-23.

[8] P. Lesaint And P.-A. Raviart, On a finite element method for solving the neutron transport equation, in Mathematical Aspects of Finite Elements in Partial Differential Equations, C.A. deBoor, ed., Academic Press, New York, 1974, pp. 89123. 